\title{
EXTENSION OF HYPERFUNCTION SOLUTIONS OF LINEAR DIFFERENTIAL EQUATIONS WITH CONSTANT COEFFICIENTS
}

\author{
TAKAHIRO KAWAI ${ }^{1}$
}

\begin{abstract}
We prove the local extensibility across a $k$-convex hypersurface of solutions of a system of linear differential equations with constant coefficients which satisfies some algebraic conditions determined by $k$. A global extensibility is also proved in the case of completely $k$-convex set.
\end{abstract}

The purpose of this note is to prove under some algebraic conditions on a system $L$ of linear differential equations with constant coefficients the (local) extensibility of hyperfunction solutions of system $L$ across a hypersurface which enjoys some "convexity" property, whose precise meaning will be given in Definition 4. Our results make a contrast to the original Hartogs-type theorems of Ehrenpreis on removable singularities of solutions of linear differential equations with constant coefficients in the sense that our extension is achieved out of a "convex" open set, not into it. In fact, what we need is the underdetermined character of system $L$ involved, not the overdetermined character, which the original result of Ehrenpreis requires. One important point in our arguments is that the flabbiness of the sheaf of hyperfunctions (Sato [8]) enables us to extend solutions even locally in spite of the fact that such an extension is not unique. See Ehrenpreis [1], Palamodov [7], Malgrange [6], Komatsu [5] and references cited there for Hartogs-type extension theorems and related results for linear differential equations with constant coefficients. See Kawai [3] for the case of linear differential equations with variable coefficients.

The main tool in our proof is the vanishing theorem for cohomology groups on a completely $(n-k)$-convex set which have as their coefficients the hyperfunction solution sheaf of a system of linear differential equations with constant coefficients (Kawai [2]). In formulating the algebraic conditions on $L$ we also use modules $E_{i}(L)(i=0,1,2, \ldots)$ introduced and fully investigated by Palamodov [7, Part II, Chapter VIII, §13].

The author would like to express his heartiest thanks to Professor Eric Bedford of Courant Institute, New York University, for calling the author's

Received by the editors August 20, 1975 and, in revised form, December 12, 1975.

AMS (MOS) subject classifications (1970). Primary 35E10; Secondary 46F 15.

Key words and phrases. System of linear differential equations with constant coefficients, $k$-convex set, hyperfunctions, flabbiness, functor $\operatorname{Hom}(M, \cdot)$.

${ }^{1}$ Supported by Miller Institute for Basic Research in Science. 
attention to the possible connection between $k$-convexity and the extendibility of solutions of a general system of linear differential equations with constant coefficients. The author also wishes to thank Professor Louis Nirenberg of Courant Institute for his kind invitation to the Institute, which made it possible for the author to discuss this problem with Professor Bedford.

In this note we mainly use the same notations as in Palamodov [7] and Komatsu [5]. One important difference from Palamodov [7] is that we denote, after Komatsu [5], by $A$ the ring $\mathbf{C}\left[\xi_{1}, \ldots, \xi_{n}\right]$ of polynomials in $n$ indeterminates $\xi_{1}, \ldots, \xi_{n}$ with coefficients in $\mathbf{C}$, though Palamodov [7] uses $9 ?$ instead of $A$. This is because we often use $\varphi p$ to denote the sheaf of pseudo-differential operators.

We first list some definitions needed to state our theorems.

Definition 1 (Palamodov [7, Part II, Chapter VIII, §11]). A real-valued $C^{2}$-function $h(x)$ defined on an open set $\Omega \subset \mathbf{R}^{n}$ is said to be $k$-convex $(1 \leqslant k \leqslant n)$ if and only if its Hessian matrix Hess $h=\left(\partial^{2} h / \partial x_{i} \partial x_{j}\right)_{1 \leqslant i, j \leqslant n}$ has at least $k$ positive eigenvalues.

Definition 2 (Palamodov [7, Part II, Chapter VIII, §11]). An open set $\Omega \subset \mathbf{R}^{n}$ is said to be $k$-convex if and only if there exists a continuous function $h(x)$ defined on $\Omega$ which satisfies the following two conditions:

(1) There exists a compact set $K \subset \Omega$ such that $h(x)$ is $k$-convex on $\Omega-K$.

(2) For any real number $c$ the set $K_{c}=\{x \in \Omega ; h(x) \leqslant c\}$ is compact.

REMARK 1 . In this note we call $h(x)$ a norm function associated with the $k$-convex set $\Omega$.

Definition 3 (Palamodov [7, Part II, Chapter VIII, §11]). An open set $\Omega \subset \mathbf{R}^{n}$ is said to be completely $k$-convex if and only if $\Omega$ is $k$-convex and the exceptional set $K$ in condition (1) is void.

Definition 4. An open set $\Omega \subset \mathbf{R}^{n}$ is said to be locally $k$-convex at $x^{0} \in \partial \Omega$, the boundary of $\Omega$, if and only if there exists an open neighborhood $U$ of $x^{0}$ such that $U \cap \Omega$ is $k$-convex.

Aside from these geometrical notions, we also need the notion of $A$-module $E_{i}(L)$ defined for $A$-module $L$ and $i=0,1,2, \ldots$, which has been introduced by Palamodov [7, Part II, Chapter VIII, $\left.\S 13,4^{0}\right]$. They are defined as follows:

$$
E_{0}(L)=\operatorname{Ker} j_{L}
$$

Here $j_{L}$ denotes the canonical map from $L$ to $\operatorname{Hom}(\operatorname{Hom}(L, A), A)$.

$$
\begin{gathered}
E_{1}(L)=\operatorname{Coker} j_{L}, \\
E_{i}(L)=\operatorname{Ext}^{i-1}(\operatorname{Hom}(L, A), A), \quad i=2,3, \ldots
\end{gathered}
$$

We use these modules to express our theorems in an intrinsic manner.

Note further that Palamodov [7] has also given a nice geometrical sufficient condition for the vanishing of these modules. See Corollary 4 in Part II, Chapter VII, $\S 13,5^{0}$ of Palamodov [7].

REMARK 2 . If $\Omega$ is $k$-convex, then $\Omega$ is locally $k$-convex at any $x^{0} \in \partial \Omega$. In order to see this, we first choose an open set $U=\left\{x \in \mathbf{R}^{n} ;\left|x_{j}-x_{j}^{0}\right|<\varepsilon\right.$, 
$j=1, \ldots, n\}(\varepsilon>0)$ and associated norm function

$$
h_{U}(x)=\sum_{j=1}^{n} \frac{1}{\varepsilon^{2}-\left(x_{j}-x_{j}^{0}\right)^{2}} .
$$

By making use of a norm function $h(x)$ associated with $\Omega$, we define

$$
\tilde{h}(x)=h(x)+h_{U}(x) .
$$

It is readily seen that $\tilde{h}(x)$ serves a norm function associated with $U \cap \Omega$ for sufficiently small $\varepsilon$ so that $U \cap \Omega$ becomes $k$-convex, since Hess $h_{U}(x)$ is positive definite there.

Note that $U \cap \Omega$ can be chosen to be completely $k$-convex. Note also that it is always the case, that is, if $\Omega$ is locally $k$-convex at $x^{0} \in \partial \Omega$, then we can find an open neighborhood $V$ of $x^{0}$ so that $V \cap \Omega$ is completely $k$-convex.

REMARK 3. Let $\varphi(x)$ be a real-valued $C^{2}$-function defined on an open set $V \subset \mathbf{R}^{n}$. Let $\Omega$ be an open set defined by $\{x \in V ; \varphi(x)<0\}$. If Hess $\varphi$ has at least $k$ positive eigenvalues at $x^{0} \in \partial \Omega$, then $\Omega$ is locally $k$-convex at $x^{0}$. In fact, choosing coordinate system $\left(x^{\prime}, x^{\prime \prime}\right) \in \mathbf{R}^{k} \times \mathbf{R}^{n-k}$ suitably, we may assume that Hess $_{x^{\prime}} \varphi(x)$ is positive definite on a sufficiently small neighborhood $V$ of $x^{0}$. Therefore

$$
\operatorname{Hess}_{x^{\prime}}\left(-\frac{1}{\varphi(x)}\right)=\left(\frac{\partial^{2} \varphi / \partial x_{i} \partial x_{j}}{\varphi^{2}}-\frac{2\left(\partial \varphi / \partial x_{i}\right)\left(\partial \varphi / \partial x_{j}\right)}{\varphi^{3}}\right)_{1<i, j<k}
$$

is positive definite on $V \cap \Omega$, since

$$
\sum_{i, j=1}^{k} \frac{\partial \varphi}{\partial x_{i}} \frac{\partial \varphi}{\partial x_{j}} \xi_{i} \xi_{j}=\left(\sum_{i=1}^{k} \frac{\partial \varphi}{\partial x_{i}} \xi_{i}\right)^{2} \geqslant 0
$$

holds for any real vector $\xi=\left(\xi_{1}, \ldots, \xi_{k}\right)$. Then, choosing $U$ and $h_{U}(x)$ as in the previous remark, we clearly see that $-1 / \varphi(x)+h_{U}(x)$ serves as a norm function of a completely $k$-convex set $U \cap \Omega$. This proves that $\Omega$ is locally $k$-convex at $x^{0}$.

Now we state our theorems.

THEOREM 1. Let an open set $\Omega \subset \mathbf{R}^{n}$ be locally $(n-k)$-convex at $x^{0} \in \partial \Omega$. Let $L$ be a system of linear differential equations with constant coefficients defined on $\mathbf{R}^{n}$ which satisfies the condition

$$
E_{i}(L)=0, \quad i=0, \ldots, k .
$$

Then, for any hyperfunction solution $u(x)$ of system $L$ defined on $V \cap \Omega$ for an open neighborhood $V$ of $x^{0}$ in $\mathbf{R}^{n}$, we can find an open neighborhood $U$ of $x^{0}$ in $\mathbf{R}^{n}$ and a hyperfunction solution $\tilde{u}(x)$ of system $L$ defined on $U \cup(V \cap \Omega)$ so that it coincides with $u(x)$ on $V \cap \Omega$.

Proof. Theorem 3 in Part II, Chapter VIII, $\$ 13$ of Palamodov [7] says that we can find an exact sequence of the following form (8) under condition (7):

$$
0 \rightarrow L \rightarrow A^{s_{k}} \stackrel{q_{k-1}}{\rightarrow} A^{s_{k-1}} \stackrel{q_{k-2}}{\rightarrow} \cdots \stackrel{q_{1}}{\rightarrow} A^{s_{1}} \stackrel{q_{0}}{\rightarrow} A^{s_{0}} .
$$


Here $q_{j}(j=0, \ldots, k-1)$ denotes a matrix of polynomials of size $s_{j-1} \times s_{j}$.

If we define $A$-module $M$ by $A^{s_{0}} / q_{0} A^{s_{1}}$, then the exact sequence (8) clearly implies that there exists the following isomorphism (9) for any $A$-module $\Phi$ :

$$
\operatorname{Ext}^{i}(L, \Phi)=\operatorname{Ext}^{k+1+i}(M, \Phi) \text { for any } i \geqslant 1 \text {. }
$$

On the other hand, the local $(n-k)$-convexity of $\Omega$ at $x^{0}$ implies the existence of a convex open neighborhood $U$ of $x^{0}$ in $\mathbf{R}^{n}$ so that $U \subset V$ and that $U \cap \Omega$ becomes a completely $(n-k)$-convex set. (See Remark 2.) Therefore we can apply Theorem 1 of Kawai [2] to assert that

$$
\operatorname{Ext}^{j}(M, \mathscr{B}(U \cap \Omega))=0 \text { for } j \geqslant k+1 \text {. }
$$

Here we have used the same notation as in Palamodov [7]. The announcement of Kawai [2] uses $\operatorname{Ext}_{\mathscr{D}}^{j}(U \cap \Omega ; M$, 饮 $)$ instead of $\operatorname{Ext}^{j}(M, \mathscr{G}(U \cap \Omega)$ ). The symbol $\mathscr{B}(U \cap \Omega)$ denotes the space of hyperfunctions defined on $U \cap \Omega$. The corresponding vanishing theorem for cohomology groups on completely $(n-k)$-convex set is not known without the assumption of hypoellipticity of $M$ if we replace $\mathscr{B}(U \cap \Omega)$ by $(\mathcal{D})^{\prime}(U \cap \Omega)$, the space of distributions on $U \cap \Omega$ (cf. Theorem 1 and Corollary 2 in Part II, Chapter VII, $\S 11$ of Palamodov [7]), while $M$ is not hypoelliptic in general in our case. Here appears one of the advantages in discussing the extension of hyperfunction solutions, not that of distribution solutions.

In passing, let us consider the following long exact sequence (11), whose existence is an immediate consequence of the flabbiness of sheaf $\mathscr{B}$ of hyperfunctions as is shown below. (See Sato [8] and the exposition [5] of Komatsu for the flabbiness of sheaf $\mathscr{B}$.) Here again appears the evidence of the advantages in discussing the extension of hyperfunction solutions.

$$
\begin{aligned}
\ldots & \rightarrow \operatorname{Ext}^{j}\left(M, \mathscr{B}_{U-(U \cap \Omega)}(U)\right) \rightarrow \operatorname{Ext}^{j}(M, \mathscr{G}(U)) \\
& \rightarrow \operatorname{Ext}^{j}(M, \mathscr{G}(U \cap \Omega)) \rightarrow \operatorname{Ext}^{j+1}\left(M, \mathscr{G}_{U-(U \cap \Omega)}(U)\right) \\
& \rightarrow \operatorname{Ext}^{j+1}(M, \mathscr{B}(U)) \rightarrow \ldots
\end{aligned}
$$

Here $\mathscr{P}_{U-(U \cap \Omega)}(U)$ denotes the space of hyperfunctions defined on $U$ with support in $U-(U \cap \Omega)$, the complementary set of $U \cap \Omega$ in $U$, which is closed in $U$. In fact, the flabbiness of sheaf 93 of hyperfunctions implies the existence of the following short exact sequence:

$$
0 \rightarrow \Re_{U-(U \cap \Omega)}(U) \rightarrow \Re(U) \rightarrow \mathscr{B}(U \cap \Omega) \rightarrow 0 .
$$

Then the long exact sequence (11) is immediately obtained by applying the functor $\operatorname{Hom}(M, \cdot)$ to the short exact sequence (11).

Since $U$ is convex by the assumption, we have

$$
\operatorname{Ext}^{j}(M, \mathscr{B}(U))=0 \text { for } j \geqslant 1 \text { (Theorem } 3 \text { of Komatsu [4]). }
$$

Therefore (10) combined with (11) and (13) implies

$$
\operatorname{Ext}^{j}\left(M, \Re_{U-(U \cap \Omega)}(U)\right)=0 \text { for } j \geqslant k+2 .
$$

Note that $k \geqslant 0$ by the assumption. Therefore, applying (9) by choosing $\mathscr{P}_{U-(U \cap \Omega)}(U)$ as $\Phi$, we conclude that 


$$
\operatorname{Ext}^{i}\left(L, \Re_{U-(U \cap \Omega)}(U)\right)=0 \text { for } i \geqslant 1 .
$$

On the other hand, applying the functor $\operatorname{Hom}(L, \cdot)$ to (12), we find the long exact sequence:

$$
\begin{aligned}
0 & \rightarrow \operatorname{Ext}^{0}\left(L, \mathscr{B}_{U-(U \cap \Omega)}(U)\right) \rightarrow \operatorname{Ext}^{0}(L, \mathscr{B}(U)) \\
& \rightarrow \operatorname{Ext}^{0}(L, \mathscr{B}(U \cap \Omega)) \rightarrow \operatorname{Ext}^{1}\left(L, \mathscr{B}_{U-(U \cap \Omega)}(U)\right) \rightarrow \ldots
\end{aligned}
$$

Then (15) implies that

$$
\begin{aligned}
& \operatorname{Ext}^{0}(L, \mathscr{B}(U)) \rightarrow \operatorname{Ext}^{0}(L, \mathscr{S}(U \cap \Omega)) \rightarrow 0 \\
& \text { is exact. }
\end{aligned}
$$

This is equivalent to saying that for any hyperfunction solution $u(x)$ of system $L$ defined on $U \cap \Omega$ we can find a hyperfunction solution $u(x)$ of $L$ defined on $U$. This ends the proof of Theorem 1 .

By making use of the notion of complete $k$-convexity, it is easy to globalize Theorem 1 as follows:

THEOREM 2. Let an open set $\Omega \subset \mathbf{R}^{n}$ be completely $(n-k)$-convex. Let $L$ be a system of linear differential equations with constant coefficients defined on $\mathbf{R}^{n}$ which satisfies condition (7). Then, for any hyperfunction solution $u(x)$ of system $L$ defined on $\Omega$, we can find a hyperfunction solution $\tilde{u}(x)$ of system $L$ defined on $\mathbf{R}^{n}$ so that it coincides with $u(x)$ on $\Omega$.

Proof. Define $A$-module $M$ as in the proof of Theorem 1 by making use of long exact sequence (8), whose existence is guaranteed by condition (7). Then the complete ( $n-k)$-convexity of $\Omega$ implies by Theorem 1 of Kawai [2] that

$$
\operatorname{Ext}^{j}(M, \mathscr{G}(\Omega))=0 \text { for } j \geqslant k+1 \text {. }
$$

We also know

$$
\operatorname{Ext}^{j}\left(M, \Re\left(\mathbf{R}^{n}\right)\right)=0 \text { for } j \geqslant 1,
$$

since $\mathbf{R}^{n}$ is convex (Theorem 3 of Komatsu [4]).

On the other hand we can find the following long exact sequence (20) by applying the functor $\operatorname{Hom}(M, \cdot)$ to the short exact sequence (19), whose existence immediately follows from the flabbiness of sheaf $\mathscr{T}$ of hyperfunctions.

$$
0 \rightarrow \mathscr{B}_{\mathbf{R}^{n}-\Omega}\left(\mathbf{R}^{n}\right) \rightarrow \mathscr{B}\left(\mathbf{R}^{n}\right) \rightarrow \mathscr{B}(\Omega) \rightarrow 0 .
$$

Here $\mathscr{B}_{\mathbf{R}^{n}-\Omega}\left(\mathbf{R}^{n}\right)$ denotes the space of hyperfunctions on $\mathbf{R}^{n}$ supported by $\mathbf{R}^{n}-\Omega$, the complementary set of $\Omega$.

$$
\begin{aligned}
\ldots & \rightarrow \operatorname{Ext}^{j}\left(M, \mathscr{B}_{\mathbf{R}^{n}-\Omega}\left(\mathbf{R}^{n}\right)\right) \rightarrow \operatorname{Ext}^{j}\left(M, \mathscr{B}\left(\mathbf{R}^{n}\right)\right) \rightarrow \operatorname{Ext}^{j}(M, \mathscr{B}(\Omega)) \\
& \rightarrow \operatorname{Ext}^{j+1}\left(M, \mathscr{B}_{\mathbf{R}^{n}-\Omega}\left(\mathbf{R}^{n}\right)\right) \rightarrow \operatorname{Ext}^{j+1}\left(M, \mathscr{B}\left(\mathbf{R}^{n}\right)\right) \rightarrow \ldots
\end{aligned}
$$

Then (17) and (18) combined with (20) imply

$$
\operatorname{Ext}^{j}\left(M, \mathscr{G}_{\mathbf{R}^{n}-\Omega}\left(\mathbf{R}^{n}\right)\right)=0 \text { for } j \geqslant k+2 .
$$

There, applying (9) by choosing ${ }^{G} \mathbf{R}^{n}-\Omega\left(\mathbf{R}^{n}\right)$ as $\Phi$, we conclude that 


$$
\operatorname{Ext}^{i}\left(L, \mathscr{B}_{\mathbf{R}^{n}-\Omega}\left(\mathbf{R}^{n}\right)\right)=0 \text { for } i \geqslant 1
$$

Therefore we immediately see that

$$
\operatorname{Ext}^{0}\left(L, \mathscr{S}\left(\mathbf{R}^{n}\right)\right) \rightarrow \operatorname{Ext}^{0}(L, \mathscr{G}(\Omega)) \rightarrow 0
$$

is exact. Thus any hyperfunction solution $u(x)$ of system $L$ defined on $\Omega$ admits an extension to $\mathbf{R}^{n}$. This ends the proof of Theorem 2 .

\section{REFERENCES}

1. L. Ehrenpreis, Fourier analysis in several complex variables, Interscience, New York, 1970. MR 44 \#3066.

2. T. Kawai, Vanishing of cohomology groups on completely $k$-convex sets, Publ. Res. Inst. Math. Sci. 11 (1975/76). (Theorem 1 of this paper has been announced in T. Kawai, Theorems on the finite-dimensionality of cohomology groups. IV, Proc. Japan Acad. 49 (1973), 655-658. MR 49 \#11574.)

3. Removable singularities of solutions of systems of linear differential equations, Bull. Amer. Math. Soc. 81 (1975), 461-463.

4. H. Komatsu, Resolutions by hyperfunctions of sheaves of solutions of differential equations with constant coefficients, Math. Ann. 176 (1968), 77-86. MR 37 \#1771.

5. , Relative cohomology of sheaves of solutions of differential equations, Lecture Notes in Math., vol. 287, Springer, Berlin and New York, 1973, pp. 192-261.

6. B. Malgrange, Systemes différentielles à coefficients constants, Séminaire Bourbaki, 1962/63, Exposé 246, Benjamin, New York and Amsterdam, 1966.

7. V. P. Palamodov, Linear differential operators with constant coefficients, "Nauka”, Moscow, 1967; English transl., Grundlehren math. Wiss., Bd. 168, Springer-Verlag, Berlin and New York, 1970; Japanese transl., Yoshioka, 1973. MR 39 \# 4517; 41 \# 8793.

8. M. Sato, Theory of hyperfunctions. II, J. Fac. Sci. Univ. Tokyo Sect. I 8 (1960), 387-437. MR 24 \# A2237.

Department of Mathematics, University of California, Berkeley, California 94720

Research Institute for Mathematical Sciences, Kyoto University, Kyoto, Japan (Current address) 